Ann. Zootech., I970, 19 (3), 255-268.

\title{
INFLUENGE DE LA TENEUR EN EAU ET DE LA DÉSHYDRATATION DE L'HERBE SUR SA VALEUR ALIMENTAIRE POUR LES VACHES LAITIERES
}

\author{
R. VÉRITÉ et M. JOURNET \\ avec la collaboration technique de Jeanne Flechet, Renée Lefaivre, \\ B. Marguis et A. Ollier \\ Station de Recherches sur l'Élevage des Ruminants, \\ Centre de Recherches de Clermont-Ferrand, 63-Saint-Genès-Champanelle \\ Institut national de la Recherche agronomique
}

\section{RÉSUMÉ}

Deux lots de ro vaches laitières, alimentées individuellement et à volonté, ont reçu à l'auge, l'un de l'herbe verte (I2 à 22 p. roo de M. S.) et l'autre la même herbe déshydratée à basse température. Six vaches munies d'une fistule du rumen ont été utilisées pour mesurer les modifications des phénomènes digestifs provoquées par la déshydratation.

La quantité de M. S. $(y)$ d'herbe verte ingérée augmente avec la teneur en M. S. $(x)$ : $y=0,208 x+9,66, v=0,9^{\mathrm{I}}{ }^{* *}$. Elle augmente également après déshydratation mais seulement lorsque 1'herbe a une teneur en M. S. inférieure à $\mathbf{r} 8$ p. roo. La consommation totale d'eau par $\mathrm{kg}$ de M. S. ingérée diminue d'environ I5 p. Ioo à la suite de la déshydratation. La digestibilité a été peu modifiée et la valeur énergétique nette mesurée par la production de lait et le gain de poids vif n'a pas varié.

La déshydratation de l'herbe a entraîné peu de modifications des phénomènes digestifs sauf en ce qui concerne le débit de liquide à l'orifice réticulo-omasal qui a diminué de plus de $30 \mathrm{p}$. Iоo.

D'un point de vue pratique, il semble possible d'extrapoler à l'herbe déshydratée longue les résultats de quantités ingérées acquis sur l'herbe verte à condition cependant que la teneur en M. S. de 1'herbe ne soit pas trop faible.

\section{IN'TRODUCTION}

Les fourrages déshydratés intéressent actuellement beaucoup d'éleveurs. Or, si l'on sait que le fourrage déshydraté (ou le foin) broyé et aggloméré est ingéré en quantité beaucoup plus importante $(+32$ à +69 p. IOO; DEMARQUILLY et JOURNET, I967) que le même produit sous forme longue, on connaît mal l'influence de la déshydratation seule sur l'acceptabilité de 1'herbe. 
D'autre part, les animaux au pâturage consomment parfois une herbe très aqueuse (en particulier au printemps) et on peut se demander si la quantité élevée d'eau qu'ils ingèrent alors n'est pas un facteur limitant de leur consommation de matière sèche. Les méthodes utilisées pour vérifier ce point ont consisté :

- soit à suivre les variations de quantités ingérées d'un même fourrage à différents stades végétatifs (donc à différentes teneurs en eau) comme l'ont fait Ducksworth et Shirlaw (I958), Hali,EY et Dougal, (I962), Demarquility (I966), Holmes et LANG ( 1963 ) qui trouvent tous (sauf HoLmes et LANG) une corrélation positive et significative entre la quantité de matière sèche ingérée et la teneur en matière sèche de l'herbe ; mais cette liaison n'est peut-être que la conséquence du vieillissement de la plante ;

- soit à comparer les quantités d'herbe verte ingérées avec celles de la même plante déshydratée (ou préfanée) en supposant que les modifications dues au traitement se limitent à l'eau. Ducksworth et ShIRLAw (I958), DAviEs (I962), HeANEY et al. (I966), MrNson (I966) n'observent pas d'action de l'eat1 par cette méthode. Cependant, beaucoup de ces expériences ont été réalisées avec très peu d'animaux ou pendant des temps très courts.

Le but de cette expérience a été d'étudier l'effet de la déshydratation en ellemême et le rôle de la teneur en eau sur l'ingestion de fourrage vert par des vaches laitières. Nous avons utilisé pour cela les 2 méthodes exposées ci-dessus et nous avons enregistré parallèlement les phénomènes digestifs sur des vaches munies d'une fistule du rumen.

\section{MATÉRIEL ET MÉTHODES}

\section{Essai d'alimentation}

Au cours d'une période de I 8 semaines (24-6 au 25-Io-68) deux lots de Io vaches laitières (Frisonnes Pie-Noire) ont reçu au même moment, l'un de l'herbe verte (lot I) et l'autre (lot II) la même herbe déshydratée non conditionnée. Ce fourrage était offert à volonté (ro p. roo de refus au moins) et constituait le seul aliment de la ration.

Les vaches étaient dans la $2^{\mathrm{e}}$ moitié de leur $2^{\mathrm{e}}$ ou $3^{\mathrm{e}}$ lactation et avaient été appariées en tenant compte des quantités de matière sèche ingérées et du niveau de production laitière enregistrés au cours d'une période préexpérimentale de 3 semaines sur un régime d'herbe seule. Respectivement pour les lots I et II la production laitière moyenne, le poids vif moyen et la

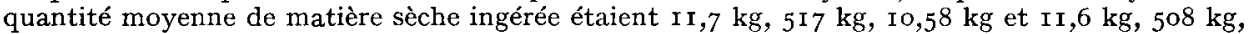
$10,47 \mathrm{~kg}$. Aucune vache n'a été utilisée après le $7^{\mathrm{e}}$ mois de gestation ce qui a nécessité au cours de la $12^{\mathrm{e}}$ semaine un changement de 5 animaux en même temps qu'un réappariement. Les valeurs pour les mêmes critères d'appariement ont alors été respectivement de $8,0 \mathrm{~kg}, 5^{\mathrm{I}} 4 \mathrm{~kg}, \mathrm{I}_{3,54} \mathrm{~kg}$ et de $7,9 \mathrm{~kg}, 524 \mathrm{~kg}, 13,50 \mathrm{~kg}$.

Treize fourrages d'espèces ou de numéros de cycles différents ont été utilisés (tabl. I). Ce sont surtout des graminées pures ou des herbes de prairies naturelles. L'herbe a été récoltée à un stade jeune, exception faite de la luzerne et du ray-grass d'Italie utilisé pendant les 2 dernières périodes, ce qui explique pour une bonne part les faibles teneurs en matière sèche (I 2 à 22 p. IOo), les teneurs moyennes en cellulose brute et les digestibilités élevées. Aucune des parcelles utilisées n'avait été pâturée précédemment dans l'année. Pour le lot I, l'herbe était fauchée chaque matin à la barre de coupe et ramassée immédiatement. Pour le lot II, de l'herbe de la même parcelle était récoltée tous les 2 jours avec une ensileuse à couteaux et déshydratée dans un séchoir Scolari à basse température $\left(150\right.$ à $\left.200^{\circ} \mathrm{C}\right)$. L'herbe verte était distribuée sous forme longue et l'herbe déshydratée sous forme de brins de 5 à $15 \mathrm{~cm}$ de longueur.

Les vaches étaient en stabulation entravée, sur litière de paille; elles recevaient le fourrage individuellement et à volonté, en 3 repas par jour $(8 \mathrm{~h} 30,15 \mathrm{~h} \mathrm{30}, \mathrm{I} 8 \mathrm{~h} \mathrm{30}$ ) et avaient en permanence de l'eau à leur disposition. Elles ont eu accès chaque jour entre i I $h$ 30 et i $5 \mathrm{~h}$ à une aire d'exercice sans herbe ni eau. 
HERBE VERTE ET DÉSHYDRATÉE

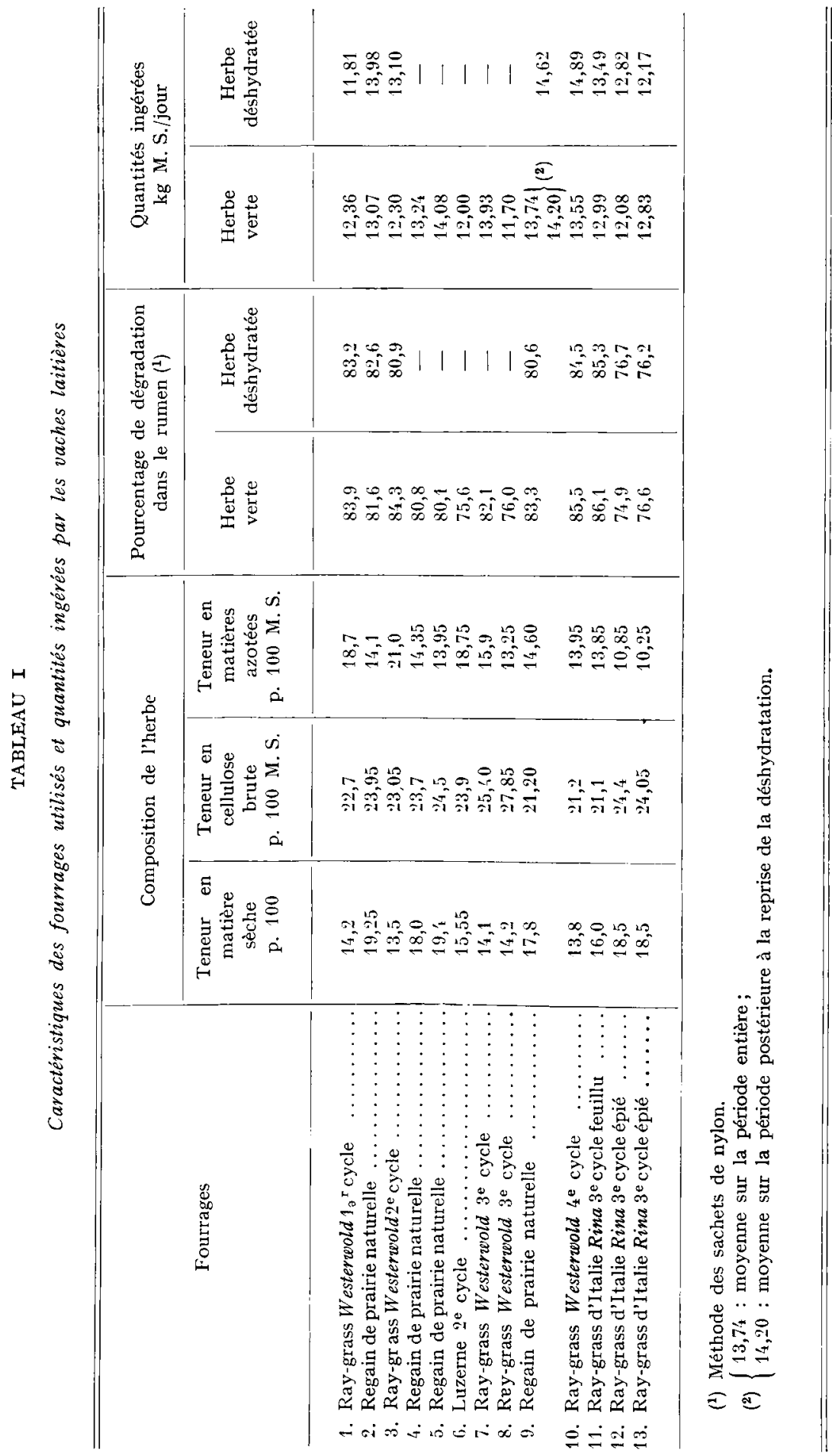


Les différentes mesures effectuées pour chaque animal et leur fréquence ont été les suivantes :

- quantité d'herbe offerte et refusée : chaque jour sauf samedis et dimanches,

- teneur en matière sèche des refus (étuve $80^{\circ}$ ) : moyenne pour le lot, une fois par jour, sauf samedis et dimanches,

- quantité d'eau bue : moyenne journalière pour chaque lot,

- quantité de lait : à chaque traite, 2 traites,

- teneur en matière grasse du lait (Gerber) : chaque jour sur un échantillon moyen des

- teneur en matières azotées totales du lait (noir amidé) : chaque semaine sur un échantillon moyen des 4 traites du mercredi et du jeudi,

- poids vif : pesée simple le jeudi, triple pesée au début et à la fin de l'expérience,

- comportement alimentaire obtenu à partir de l'enregistrement des mouvements des mandibules; pendant 4 périodes de 5 jours sur 5 vaches de chaque lot.

On a également mesuré sur une vache fistulisée la dégradation au bout de 48 heures dans le rumen des 13 fourrages par la méthode des sachets de nylon (JouRnet et DEMARQUILLY, I967).

Une panne de séchoir entre les $4^{\mathrm{e}}$ et $\mathrm{I}^{\mathrm{e}}$ semaines a réduit à $\mathrm{I}$ o semaines et demie la durée de l'expérience sur le lot I. De plus, pour être sûrs que les vaches ont bien mangé à volonté, nous avons éliminé les journées au cours desquelles 3 vaches ou plus avaient moins de ro p. roo de refus, si bien que l'interprétation a été faite sur 69 et 39 données journalières pour les lots I et II.

\section{Animaux fistulisés}

Trois fourrages sous 2 formes de présentation ont été distribués chacun à un groupe de 2 vaches munies d'une large fistule du rumen.

Chaque groupe recevait pendant 3 semaines l'herbe verte puis l'herbe déshydratée de même origine au cours des 3 semaines suivantes. Le fourrage était déshydraté en une seule fois au moment où s'effectuaient les principales mesures avec l'herbe verte. Les vaches étaient sur stalles courtes avec tapis de caoutchouc et étaient alimentées dans les mêmes conditions que les animaux en lot.

Au cours des ro derniers jours de chaque période les mesures suivantes ont été effectuées :

- digestibilité du fourrage par collecte totale des fèces ( 7 jours),

— temps de séjour des particules indigestibles (BALCH, I950),

- débit de liquide à travers l'orifice réticulo-omasal par la méthode de l'infusion continue d'une solution de polyéthylène glycol 4000 pendant 30 heures avec prélèvement horaire pendant les 2 dernières heures,

- activité cellulolytique du jus de rumen mesurée par la dégradation de paille d'avoine dans des sachets de nylon mis dans le rumen pendant $4^{8}$ heures (Journet et Demarguilly, I967),

- activité fermentaire mesurée par la quantité de gaz produite in vitro par l'incubation de contenu de rumen (Journet et DemarQuilly, I967),

- teneur en acides gras volatils (A. G. V.) du jus de rumen et composition du mélange de ces acides par chromatographie en phase gazeuse (RIGAUD et Journer, 1970),

- poids frais et sec du contenu rumino-réticulaire, 3 heures après le repas de $8 \mathrm{~h} 30$, le dernier jour.

\section{RÉSULTATS}

\section{Influence de la déshydratation sur les quantités ingérées, le comportement alimentaire et la production des animaux}

\section{Quantité de matière sèche ingérée.}

Avec l'herbe dont la teneur en matière sèche variait de 12 à $22 \mathrm{p}$. Ioo, la déshydratation a permis une augmentation limitée mais significative $(\mathrm{P}<0,05)$ des quantités ingérées par rapport à 1 'herbe verte $\left(\mathrm{I}_{3}, 62 \mathrm{~kg}\right.$ contre $\left.13,07 \mathrm{~kg}\right)$. Cette augmentation est très variable d'un jour à l'autre $(-\mathrm{I}, 72$ à $+3,83 \mathrm{~kg}$ pour une moyenne de $+0,55 \mathrm{~kg}$ ). Elle semble accrue par la présence d'eau externe à la plante verte puisqu'elle est de $0,86 \mathrm{~kg}$ dans le cas de l'herbe mouillée $(n=\mathrm{I} 8)$ et seulement 
de $0,36 \mathrm{~kg}(n=24)$ dans le cas de l'herbe sèche. Cet accroissement de la différence $(y)$ est de I $\mathrm{kg}$ quand la teneur en matière sèche $(x)$ de la plante diminue de 3 points. $y=-0,337 x+6$,Io $; r=0,8 \mathrm{I} ; n=$ Io (classes de matière sèche de I point). Ce fait est observé aussi bien avec l'herbe mouillée qu'avec l'herbe sèche.
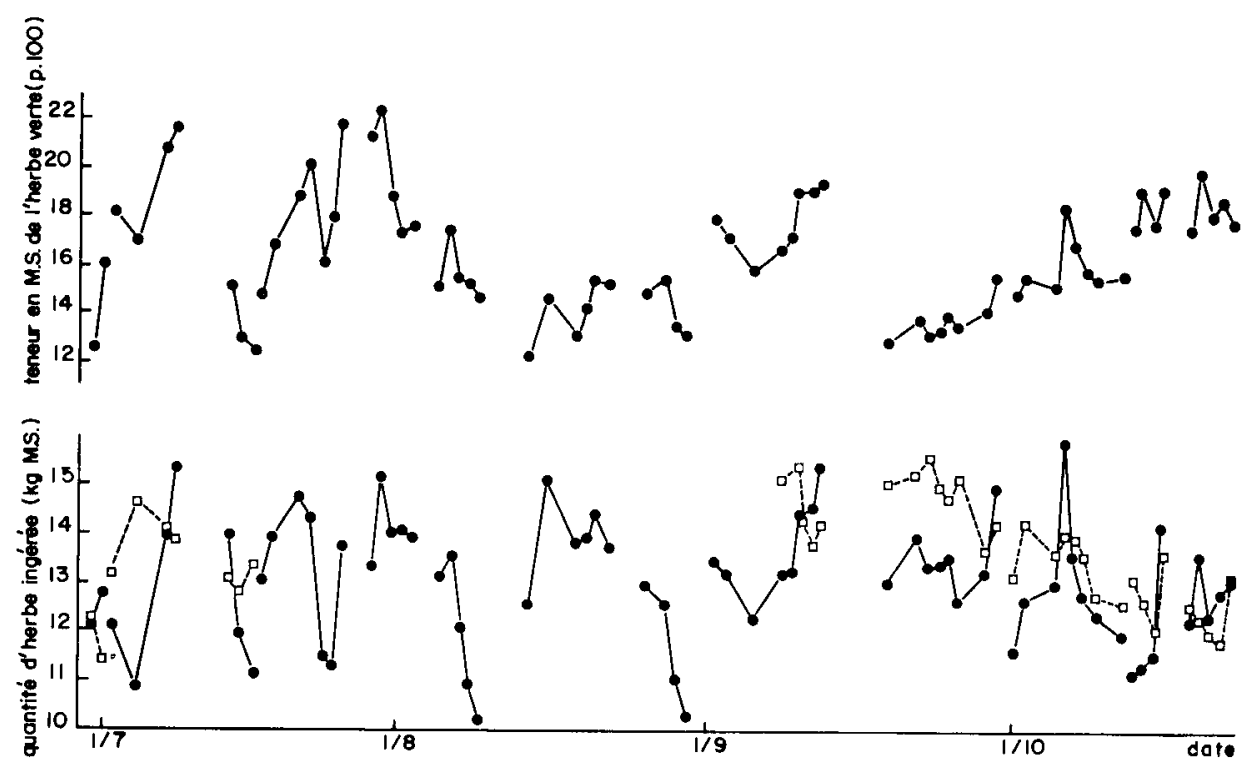

FIG. I. - Évolution journalière de la teneur en matière sèche (M. S.) de l'herbe et des quantités ingérées par les vaches laitières

$\longrightarrow$ Herbe verte

口... Herbe déshydratée

Quantité d'eau.

La quantité journalière d'eau bue par les animaux du lot recevant l'herbe verte a été en moyenne de I5,51 par animal (entre o et 401 suivant les jours), calle des animaux du lot recevant l'herbe déshydratée de 75,51 (46 à 96 1). La quantité moyenne d'eau bue par les animaux recevant de l'herbe verte a été inférieure à 51 par animal pendant II jours sur 57 ; il est probable qu'alors un certain nombre d'animaux ne buvaient pas. Cependant les animaux recevant l'herbe verte ont ingéré des quantités d'eau totale (boisson + aliments) en moyenne plus élevées : 86,5 1 (de 63 à I23 1 suivant les jours) que ceux du lot I : 76,7 1 soit respectivement 6,62 et 5,631 d'eau par $\mathrm{kg}$ de matière sèche ingérée; la différence est significative $(\mathrm{P}<\mathrm{o}$,oI $)$.

La consommation d'eau totale par $\mathrm{kg}$ de matière sèche par le lot I diminue de $I 1$ quand la teneur en matière sèche de la plante augmente de 5 à 6 points $\left(r=0,5 \mathrm{I}^{* *}\right)$.

\section{Comportement alimentaire.}

La consommation de fourrage déshydraté modifie le comportement alimentaire des animaux. La durée de consommation diminue de façon importante $(6 \mathrm{~h} 57 \mathrm{mn}$ 
contre $8 \mathrm{~h}$ I5 $\mathrm{mn}$ ) bien que la quantité ingérée soit légèrement supérieure. En revanche, le temps de rumination est plus long ( $9 \mathrm{~h} \mathrm{I2} \mathrm{mn}$ contre $8 \mathrm{~h} 36 \mathrm{mn}$ ) si bien que le temps de mastication par $\mathrm{kg}$ de matière sèche n'est pas très différent $(68 \mathrm{mn} /$ kg M. S. pour l'herbe déshydratée contre 73 pour l'herbe verte).

\section{Production.}

Seules les productions réalisées au cours de la $2^{\mathrm{e}}$ période expérimentale ont été analysées. La production laitière a augmenté au cours des 2 premières semaines car l'herbe distribuée à ce moment était de meilleure qualité que celle de la période d'appariement. L'augmentation a été plus forte pour le lot II que pour le lot I. Par la suite, la production est toujours restée plus élevée avec l'herbe déshydratée (en moyenne 8,2 contre $7,6 \mathrm{~kg}$ ). Cette diffétence correspond à une consommation plus forte d'herbe déshydratée $(+0,8 \mathrm{~kg}$ de matière sèche) que d'herbe verte bien que les 2 lots aient été suralimentés en permanence. Le taux butyreux a évolué de la même façon dans les 2 lots (baisse de I,I p. Ioo et 0,7 p. Ioo pour les lots I et II par rapport à la période préexpérimentale).

Le poids vif a évolué de la même façon dans les 2 lots. Le gain de poids vif a été de $30 \mathrm{~kg}$ en moyenne par animal, pour les 6 semaines considérées.

Pour savoir si la déshydratation modifie la valeur énergétique nette du fourrage, un bilan a été calculé pour la $2^{\mathrm{e}}$ période expérimentale à partir des résultats de production (1ait et gain de poids) et des quantités de matière sèche ingérées (tabl. 2).

TABIEAU 2

Bilan énergétique calculé à partir des résultats des 6 dernières semaines

\begin{tabular}{|c|c|c|}
\hline & Herbe verte & Herbe déshydratée \\
\hline Quantités ingérées : kg M. S./jour (1) & 12,98 & 13,78 \\
\hline Production laitière 4 p. $100: \mathrm{kg} /$ jour $\ldots \ldots \ldots$ & 8,0 & 8,5 \\
\hline Poids vif moyen $: \mathrm{kg} \quad \ldots \ldots \ldots \ldots \ldots \ldots$ & 527 & 562 \\
\hline Gain de poids $: g /$ jour $\ldots \ldots \ldots \ldots \ldots$ & 690 & 738 \\
\hline $\begin{array}{l}\text { Besoins en énergie (entretien }+ \text { production de lait } \\
\quad+\text { gain de poids) : U. F./jour ( } 2 \text { ) } \ldots \ldots \ldots \ldots\end{array}$ & $9,94_{4}^{\prime}$ & 10,47 \\
\hline Valeur énergétique nette du fourrage $\frac{(2)}{(1)}$ en & & \\
\hline U. F./kg M.S. $\ldots \ldots \ldots \ldots \ldots \ldots \ldots \ldots$ & 0,76 & 0,76 \\
\hline
\end{tabular}

Besoins d'entretion (U. F.) $=1,5+\frac{\mathrm{P}}{200} \quad \mathrm{P}=$ poids vif en $\mathrm{kg}$.

Besoins de production du lait à 4 p. 100 de matière grasse $=0,38 \mathrm{U} . \mathrm{F} . / \mathrm{kg}$.

Besoins pour le gain de poids au cours de la lactation $=4,0 \mathrm{U} . \mathrm{F} . / \mathrm{kg}$.

(C. BÉrANGER et al., 1970).

Il semble bien que cette valeur énergétique nette ne soit pas modifiée puisqu'elle est de 0,76 U. F. $/ \mathrm{kg}$ M. S. pour les 2 formes du fourrage. Cette valeur n'est qu'ind:cative car la production laitière (dont les besoins sont bien connus) est faible par rapport au gain de poids des animaux (dont les hesoins sont moins bien connus). 


\section{Variations des quantités d'herbe verte ingérées}

Matière verte.

La quantité journalière d'herbe verte ingérée par animal a été en moyenne de $80,6 \mathrm{~kg}$ pour le lot (de 56 à $106 \mathrm{~kg}$ suivant les jours). Elle a quelquefois dépassé I $20 \mathrm{~kg}$, et même $150 \mathrm{~kg}$ pour 3 animaux pendant 3 jours consécutifs de la période préexpérimentale avec une herbe à I $0,5 \mathrm{p}$. Ioo de matière sèche.

La quantité d'herbe ingérée en moyenne par animal est en liaison étroite avec la teneur en eau de la plante $(r=0,80 * *, 69$ valeurs). Elle augmente en moyenne de $4 \mathrm{~kg}$ quand la teneur en eau augmente de $I$ point. Ces résultats sont en accord avec ceux de Demarguilly (Ig66).

Matière sèche.

La quantité de matière sèche d'herbe verte ingérée a été en moyenne ( 69 jours) de $13,08 \mathrm{~kg}$ de matière sèche soit $2,5 \mathrm{I} \mathrm{kg}$ de matière sèche par Ioo $\mathrm{kg}$ de poids vif avec des extrêmes journaliers de 10,8 à $15,9 \mathrm{~kg}$ de matière sèche.

Les quantités ingérées ont varié avec le fourrage utilisé : espèce végétale, numéro de cycle, stade de végétation (tabl. I). Ainsi les repousses de prairies naturelles ont été les mieux consommées ( $3,55 \mathrm{~kg} \mathrm{M}$. S.) et le $2^{\mathrm{e}}$ cycle de luzerne le moins bien ( $12,00 \mathrm{~kg} \mathrm{M}$. S.). A digestibilité pratiquement égale, les $3^{\mathrm{e}}$ et $4^{\mathrm{e}}$ cycles de ray-

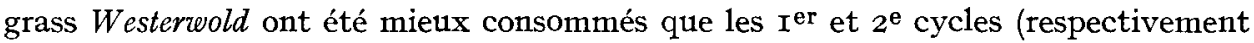
13,93 et $13,55 \mathrm{~kg}$ de M. S. contre 12,36 et $\mathrm{I} 2,30 \mathrm{~kg}$ de M. S.).

En éliminant l'influence des facteurs précédents (par comparaison des valeurs à l'intérieur de chaque période) on constate que l'humidité externe de la plante (due à la pluie ou la rosée) fait diminuer les quantités ingérées. La quantité de matière sèche ingérée avec l'herbe sèche $\left(\mathrm{r}_{3}, 45 \mathrm{~kg}\right.$ est significativement plus élevée qu'avec la même herbe mouillée $(\mathrm{I} 2,83 \mathrm{~kg})$.

De même que DeMarguilly ( $(966)$, on n'a pas trouvé de liaison entre la quantité de matière sèche ingérée et la teneur en cellulose brute de la plante mais il faut noter que les fourrages utilisés avaient tous une teneur en cellulose assez voisine (entre 20 et $26 \mathrm{p}$. Ioo de la matière sèche).

La liaison entre la quantité de matière sèche ingérée et la digestibilité mesurée par la méthode des sachets est faible $\left(r=0,65^{*}\right.$ pour I3 périodes). Ce fait peut être dû à l'hétérogénéité des espèces fourragères utilisées ou aux faibles différences existant entre les digestibilités enregistrées.

La quantité de matière sèche ingérée est liée de façon positive et significative à la teneur en matière sèche de la plante (fig. 2). Cette liaison est très élevée quand on regroupe les valeurs journalières par classes de matière sèche ( $\mathrm{I}$ point $=\mathrm{I}$ classe), $\left(r=0,9^{* *}, 9\right.$ classes). En moyenne une augmentation de la teneur en matière sèche $(x)$ de 5 points correspond à une augmentation des quantités ingérées $(y)$ de I $\mathrm{kg}$.

Cette liaison existe aussi bien avec l'herbe sèche $\left(y_{s}\right)$ qu'avec l'herbe humide $\left(y_{h}\right)$ :

$$
\begin{array}{lll}
y_{s}=0,167 x+10,59 & 4 \mathrm{I} \text { valeurs } & r=0,42 \\
y_{n}=0,232 x+8,88 & 28 \text { valeurs } & r=0,39 * *
\end{array}
$$

Quand on étudie les fourrages séparément on ne trouve de liaison significative que dans 4 cas sur les 9 étudiés par suite du petit nombre de données et de la faible 
étendue des teneurs en matière sèche. La régression est alors sensiblement différente : l'augmentation des quantités ingérées par point de teneur en matière sèche serait alors de 0,67 à $1,03 \mathrm{~kg}$ de matière sèche suivant les fourrages.

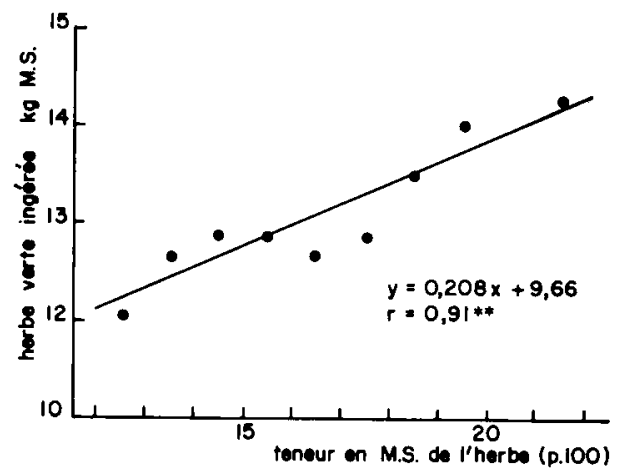

FIG. 2. - Influence de la variation de la teneur en matière sèche (M. S.) de l'herbe sur la quantité ingérée

\section{Phénomènes digestifs}

La comparaison des 2 formes de présentation a été réalisée avec 3 fourrages :

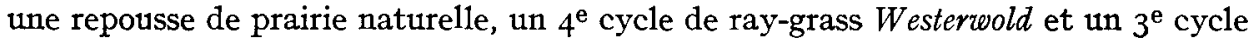
de ray-grass d'Italie Rina (tabl. 3 ).

La quantité de matière sèche ingérée sous forme d'herbe déshydratée a été plus élevée que sous forme verte pour les deux premiers fourrages étudiés $(+0,65$ et $+\mathrm{I}, 22 \mathrm{~kg}$ de matière sèche) et plus faible pour le $3^{\mathrm{e}}$ fourrage $(-0,9 \mathrm{I} \mathrm{kg}$ de matière sèche). Ces différences vont dans le même sens que celles observées sur les vaches en lot.

\section{Digestibilité.}

La digestibilité de la matière sèche obtenue par la méthode de collecte totale des fèces a été identique pout les 2 formes de présentation dans le cas des $2^{\mathrm{e}}$ et $3^{\mathrm{e}}$ fourrages étudiés; par contre, elle a été diminuée de 3 points $(69,2$ contre $72, \mathrm{I})$ par la déshydratation pour le I $^{\text {er }}$ fourrage.

Les résultats obtenus par la méthode des sachets (tabl. I) montrent également qu'en général la déshydratation diminue peu la digestibilité : $82,0 \mathrm{p}$. Ioo de dégradation pour l'herbe verte contre $8 \mathrm{I}, 2 \mathrm{p}$. Ioo pour l'herbe déshydratée avec 8 échantillons. Ces résultats sont en accord avec ceux de DEMARQUILLY sur des moutons.

\section{Contenus digestifs et vitesse de transit des particules indigestibles.}

Les poids frais et sec du contenu de rumen ont varié dans le même sens que la quantité de matière sèche ingérée mais pas de façon proportionnelle. Le poids sec des contenus a été plus élevé avec l'herbe déshydratée qu'avec l'herbe verte pour les deux premiers fourrages (de $+48 \mathrm{p}$. Ioo et $+29 \mathrm{p}$. IOo) et plus faible pour le dernier (de $-7 \mathrm{p}$. IOO) alors que les différences dans les quantités ingérées ont été respectivement de + I5 p. Ioo, $+30 \mathrm{p}$. Ioo et $-2 \mathrm{p}$. Ioo pendant les deux jours précédant les mesures. 
HERBE VERTE ET DÉSHYDRATÉE

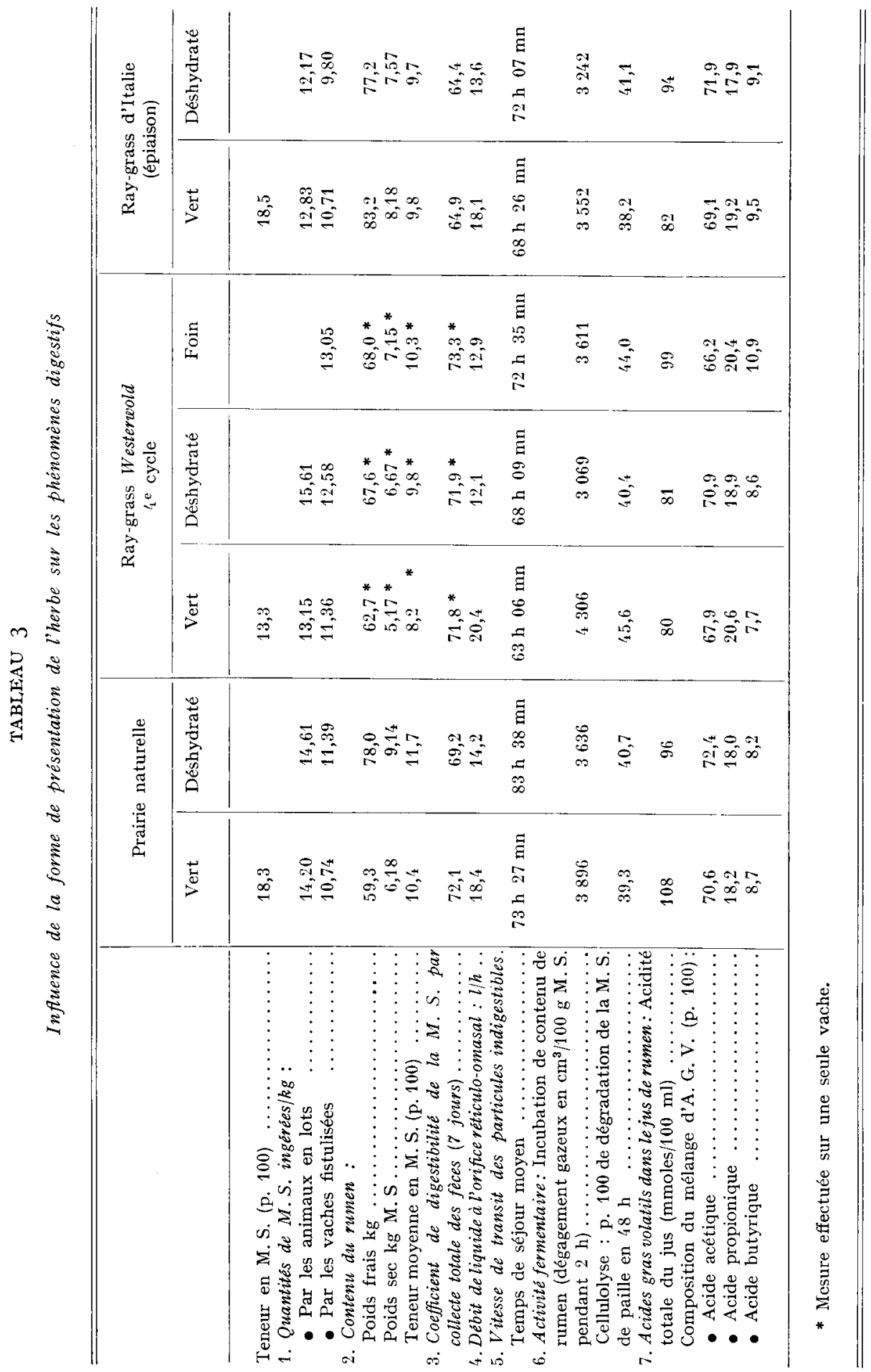


La déshydratation a surtout eu pour effet d'augmenter systématiquement la teneur en matière sèche du contenu du rumen d'environ I point en moyenne (de 9,6 à Io,6 p. Ioo M. S.). L'augmentation a surtout été sensible dans les zones habituellement les plus liquides, en particulier dans le sac ventral comme le montrent les valeurs de teneur en matière sèche du contenu en différents endroits :

$\begin{array}{cr}\begin{array}{c}\text { Herbe verte } \\ \text { (p. Ioo) }\end{array} & \begin{array}{r}\text { Herbe déshy } \\ \text { (p. Ioo) }\end{array} \\ \text { I4,3 } & - \\ \text { I I,3 } & \text { I4,9 } \\ 4,8 & \text { I3,4 } \\ \text { I3,6 } & 5,9\end{array}$

Le temps moyen de séjour des particules indigestibles est légèrement plus long avec 1 'herbe déshydratée qu'avec l'herbe verte : respectivement $74 \mathrm{~h} 30 \mathrm{mn}$ et $68 \mathrm{~h} 20 \mathrm{mn}$. Ce phénomène peut être relié aux poids de contenus plus élevés dans le cas de l'herbe déshydratée.

\section{Débit de liquide.}

Les débits de liquide à l'orifice réticulo-omasal ont toujours été très élevés (entre $\mathrm{I} 2$ et $201 / \mathrm{h}$ ); les débits obtenus avec l'herbe verte ont dépassé d'environ 40 p. Ioo ceux obtenus avec l'herbe déshydratée (I9,0 1/h contre $13,31 / \mathrm{h}$ ). Si on suppose que la quantité d'eau présente dans le rumen le jour de la mesure est la même que celle présente le jour du vidage du contenu, ces débits horaires représentent 30,3 p. Ioo et I 9,3 p. Ioo de l'eau contenue dans le rumen respectivement pour 1 'herbe verte et 1'herbe déshydratée.

\section{Activité fermentaire.}

L'activité cellulolytique du jus de rumen est identique pour les 2 formes de fourrages : 4I,o et $40,7 \mathrm{p}$. Ioo de la paille contenue dans les sachets sont dégradés en 48 heures dans le rumen des animaux qui ingèrent respectivement l'herbe verte et l'herbe déshydratée. Par contre, l'activité fermentaire mesurée au cours de l'incubation de contenu est systématiquement plus faible avec l'herbe déshydratée qu'avec 1'herbe verte, ce qui est peut-être une cause de la diminution de la vitesse de transit. La teneur du jus de rumen en A. G. V. n'est pas influencée par la forme de présentation de 1'herbe (elle est en moyenne de 90 mmoles/1) mais il semble cependant qu'il y ait une légère déviation des fermentations. En effet, la composition du mélange d'A. G. V. dans le jus de rumen est légèrement modifiée : avec l'herbe déshydratée on note une augmentation faible mais systématique de la part de l'acide acétique ( $7 \mathrm{I}, 7$ contre $69, \mathrm{I}$ p. Ioo en moyenne) aux dépens de l'acide propionique et surtout des acides valérianique et isovalérianique dans le mélange. P. LARVOR et C. VIOLETTE (I969) ont également noté l'augmentation de la part de l'acide acétique aux dépens de celle de l'acide propionique à la suite de la déshydratation de l'herbe.

\section{Comparaison avec du foin.}

Une partie de $1^{\prime}$ herbe utilisée au cours de la $2^{\mathrm{e}}$ période $\left(4^{\mathrm{e}}\right.$ cycle de ray-grass Westerwold) a été récoltée en foin séché sur le champ et distribuée aux mêmes animaux. Les quantités ingérées, les quantités de contenus et les débits horaires de 
liquides à l'orifice réticulo-omasal ont été très proches de ceux obtenus avec le même fourrage déshydraté. Par contre, la composition du mélange d'acides gras volatils du rumen est différente et ressemble plus à celle obtenue avec le fourrage vert.

\section{DISCUSSION}

Nous avons trouvé une liaison entre la teneur en matière sèche de l'herbe verte et la quantité de matière sèche ingérée. Nous avons également mis en évidence un accroissement des quantités ingérées à la suite de la déshydratation mais seulement pour une teneur en matière sèche de l'herbe inférieure à I $8 \mathrm{p}$. Ioo. Ces résultats semblent indiquer un effet dépressif de l'eau contenue dans l'herbe sur les quantités de matière sèche ingérées, cet effet ne se manifestant qu'à partir d'une certaine quantité d'eau totale ingérée, c'est-à-dire au-dessous d'un seuil de teneur en matière sèche de l'herbe comme le supposaient Ducksworth et SHIRLAW (I958). Ceci permettrait d'expliquer :

a) Les résultats discordants des différents auteurs (voir introduction) en ce qui concerne la liaison entre teneur en matière sèche et quantité ingérée. On voit en effet que cette liaison n'a été trouvée qu'avec l'herbe à teneur en matière sèche faible.

b) L'absence d'effet de la déshydratation sur les quantités ingérées notée par Ducksworth et SHiRlaw (1958), Minson (1956) et HEANEY et al. (I966) qui ont utilisé de l'herbe dont la teneur en matière sèche a toujours été supérieure à I $9 \mathrm{p}$. Ioo (au moins pour les premiers).

L'eau contenue en excès dans l'herbe verte peut réduire les quantités ingérées par son pouvoir encombrant ou en agissant sur les phénomènes digestifs et gustatifs. D'après CAMPLING et BALCH (Ig6I) le pouvoir encombrant de l'eau est nul, mais cette affirmation est basée sur un essai où l'eau était ajoutée artificiellement par une fistule du rumen et en quantité relativement normale (4,5 1 par $\mathrm{kg}$ de matière sèche ingérée).

L'importance des quantités d'eau ingérées (eau bue et eau de la plante) par kg de matière sèche par les vaches qui ont consommé de l'herbe très aqueuse, fait penser que la quantité d'eau apportée par l'herbe a dû être parfois excessive. Jusqu'à une teneur en matière sèche de l'herbe de I5 p. Ioo, les quantités d'eau bues en moyenne par animal et par jour ont été inférieures à Io 1. HALLEX-DOUGALI (I962) et MCLUSKy (I955), avec des vaches à 1'herbe, trouvent également des ingestions d'eau par $\mathrm{kg}$ de matière sèche élevées (respectivement 5 , I à 5,8 et 6,9 à 7, I $1 / \mathrm{kg}$ de matière sèche). OWEN et al. (I967) et WAL,Do $e t$ al. (I965) avec des régimes hivernaux trouvent des valeurs plus faibles (respectivement 2,6 à 4,8 et 3,2 à 4,9 $1 / \mathrm{kg}$ de matière sèche).

L'augmentation sensible des consommations due à la déshydratation a pu être reliée :

a) A des modifications du comportement alimentaire puisque le temps de consommation est considérablement diminué avec l'herbe déshydratée et il est du même ordre que celui des régimes de type hivernal ( $7 \mathrm{~h}$ or $\mathrm{mn} \pm 8,4 \mathrm{p}$. Ioo, JourNET, non publié). 
b) A des modifications au niveau digestif, en particulier les contenus secs du rumen sont nettement plus élevés dans 2 cas sur 3 et surtout les débits de liquide sont considérablement plus faibles avec de 1'herbe déshydratée. Avec 1'herbe déshydratée, les débits par rapport au volume de liquide dans le rumen sont faibles ( 7,5 à 20,5 p. IOo) et semblables à ceux observés avec des régimes hivernaux (7,5 à 22, I p. IOo) par LAMPILA (I965), AAFJES et NiJHOF (Ig69) et OWEN et al. (I968). Avec 1'herbe verte, Hutron (I964) et Tulion et al. (I965) trouvent comme nous des débits plus forts : 20 à $35 \mathrm{p}$. roo. En revanche, les autres phénomènes digestifs ne permettent pas d'expliquer l'augmentation des quantités ingérées. Au contraire, avec l'herbe verte, la vitesse de fermentation et la vitesse de transit des particules indigestibles sont plus grandes.

Malgré les modifications dans les phénomènes digestifs, il ne semble pas y avoir de variation importante de la valeur énergétique nette de l'herbe par déshydratation dans les conditions de l'expérience, d'après les résultats de production enregistrés. Cependant, EKERN et al. (I964) constatent avec des moutons que la déshydratation de $1^{\prime}$ herbe dans un four à $100^{\circ} \mathrm{C}$ provoque une augmentation de I $5 \mathrm{p}$. Ioo de la valeur énergétique nette par suite d'une diminution des pertes sous forme de chaleur. En revanche, ScHOCH et al, (I964) constatent également avec des moutons que la valeur énergétique nette de $1^{\prime}$ herbe déshydratée à $60^{\circ} \mathrm{C}$ est inférieure de $\mathrm{I} 7 \mathrm{p}$. Ioo à celle de la même herbe conservée par le froid.

Notre étude montre que la déshydratation (non suivie de conditionnement) des fourrages verts effectuée à basse température et dans de bonnes conditions modifie peu ou pas la valeur énergétique. Les quantités de matières sèches ingérées changent peu sauf si 1'herbe est très aqueuse ( $<$ I5 p. Ioo de matière sèche). En conséquence, les quantités d'herbe déshydratée (non conditionnée) ingérées peuvent varier dans de grandes limites comme celles d'herbe verte et à cause des mêmes facteurs. On sait que, par contre, le conditionnement apporte des modifications.

Reçu pour publication en juin 1970.

\title{
SUMMARY
}

\begin{abstract}
EFFECT OF THE WATER CONTENT OF GRASS AND DEHYDRATION A'T LOW TEMPERATURE UPON I'TS FEEDING VALUE FOR DAIRY COWS
\end{abstract}

I. We studied the effect of drying (dryer Scolari $150-200^{\circ} \mathrm{C}$ ) and of the water content of grass upon its intake by dairy cows.

2. During I 8 weeks, 2 lots of Io dairy cows were fed, ad libitum, fresh grass (lot I) or the same grass dried (lot II) in blades of 5 to $15 \mathrm{~cm}$ length. Characteristics and total intakes of grasses are shown table $\mathrm{I}$.

Dry matter (D. M.) intake of dried grass was significantly higher than that of fresh grass $(\Delta=0,55 \mathrm{~kg})$. The difference $(y, \mathrm{~kg}$ D. M.) between intakes (lot II - lot I) was related to the dry matter content of the fresh grass ( $x, \mathrm{p}$, Ioo).

$$
y=-0,337 \mathrm{X}+6, \mathrm{IO} \quad r=0,8 \mathrm{I} * *
$$

Water intake was significantly higher in lot I than in lot II :6,6 and 5,6 I par $\mathrm{kg}$ of D. M. intake respectively. When the animals were fed dried grass, the mean time spent daily in eating was higher, but the time spent in ruminating was longer than when they were fed fresh grass. 
The milk yields were identical and the energy values of fresh and dried grass, calculated from the amount of milk produced and the weight gain, were the same.

Fresh grass intake $(y=\mathrm{kg}$ D. M. per cow per day) was related to dry matter content $(x$, p. Ioo) of grass :

$$
y=0,208 \mathrm{X}+9,66 \quad r=0,9 \mathrm{I}^{* *}
$$

3. Digestion of 3 of the roughages given to the animals was studied with 3 groups of 2 fistulated cows receiving these forages successively fresh and dried (table 3 ).

When dried grass was fed, the mean time of retention of food residues in the gut was a little higher, while the flow of liquid through the reticulo-omasal opening was about 30 p. Ioo lower than when fresh grass was fed.

The fermentations were not modified very much : with dried grass the acetic acid content of the rumen liquor was a little lower than with fresh grass.

4. It seems that the water of the plant has a decreasing effect upon the food intake in the case of aqueous grass (less than i 8 p. I oo D. M.). However, with dairy cows, when the dry matter content of grass is higher than I 5 p. Ioo, the intake is quite the same, should the grass be fresh or dried (but not ground).

\section{RÉFÉRENCES BIBLIOGRAPHIQUES}

AafJes J. H., NijhoF J. K., I968. Measurements of flow through the cows rumen by continuous infusion of a marker. Tijdschr. Diergeneesk. deel 93, afl. 1, 16-27.

Arnold G. W., 1962. Effects of pasture maturity on the diet of sheep. Austr. J. of Agric. Res., 13, 7OI-706.

Béranger C., Negrin M., Malterre C., I97o. Évolution du gain de poids vif et de l'état d'engraissement des vaches taries au pâturage. Ann. Zootech., 19, 53-66.

Campling R. C., Balch C. C., I96r. Factors affecting the voluntary intake of food by cows. I Preli. minary observations on the effect, on the voluntary intake of hay, of changes in the amount of the reticulo-ruminal contents. Brit. J. Nutr., 15, 523-530.

Davies H. L., 1962. Intake studies in sheep involving high fluid intake. Proc. Austr. Soc. Anim. Prod., 4, I67-I7I.

Demarquilly C., I966. Valeur alimentaire de l'herbe des prairies temporaires aux stades d'exploitation pour le pâturage. II. Quantité ingérée par les vaches laitières. Ann. Zootech., 15, I47-169.

Demarquilly C., Journet M., I967. Valeur alimentaire des foins condensés. I. Influence de la nature du foin et de la finesse de broyage sur la digestibilité et la quantité ingérée. Ann. Zootech., 16, I23-I50.

Ducksworth J. E., Shirlaw D. W., I958. A study of factors affecting feed intake and the eating behaviour of cattle. Anim. Behav., VI, I47-I54.

Ekern A., Blaxter K. L., Sawers D., I964. The effect of artificial drying and of freezing on the energy value of pasture herbage. "Energy Metabolism". Proc. 3 rd Symp. Troon. Scotland. May $1964,217$.

Halley R. J., Dougall B. M., 1962. The feed intake and performance of dairy cows feed on cut grass. J. Daipy Res., 23, $24 \mathrm{I}+248$.

Heaney D. P., Piguen W. J., Pritchard G. I., ig66. The effect of freezing pasture herbage on digestibility and voluntary intake assays with sheep. Proc. Xth int. Grassl. Congr., 379・384.

Holmes J. C., Lang R. W., I963. Effects of fertiliser nitrogen and herbage dry matter content on herbage intake and digestibility in bullocks. Anim. Prod., 5, 17-26.

Hutton J. B., Hugues J. W., Newth R. P., Watanabe K., I964. The voluntary intake of the lactating dairy cow and its relation to digestion. Proc. Nz Soc. Anim. Prod., 24, 29-42.

Journet M., Demarquilly C., I967. Valeur alimentaire des foins condensés. II. Influence du broyage et de la mise en agglomérés sur la digestion du foin de luzerne dans le rumen. Ann. Zootech., 16, 307-32I.

LAmpila M., I965. The passage of fluid, certain mineral elements, and volatile fatty acids from the reticulo-rumen of the cow. Ann. Agric. Fenn, 4, r34-I 44 .

LARVOR P., VIolette C., I969. Influence de l'ingestion d'herbe tétanigène sur le métabolisme minéral $(\mathrm{Mg}, \mathrm{Ca}, \mathrm{P}, \mathrm{Na}, \mathrm{K}$ ) et certains éléments du métabolisme énergétique (corps cétoniques, acides gras volatils) chez la brebis. Rech. Vet. (2), 27-44.

Mc Lusky D.S., 1952-55. The quantities of herbage eaten by grazing dairy cows. Proc. Brit. Soc. Anim. Prod. 45-51.

Minson D. J., I966. The intake and nutritive value of fresh, frozen and dried Sorghum almum,Digitaria decumbens and Panicum maximum. J. Brit. Grassl. Soc., 21, I23-126.

OWen J. B., Miller E. L., Bridge P. S., I968. A study of the voluntary intake of food and water and the lactation performance of cows given diets of varying roughage contents ad libitum. J.Agric. Sci., $70,223-255$. 
Rigaud J., Journet M., I97o. Méthode de dosage des acides gras volatils dans le liquide du rumen. Ann. Biol. anim. Bioch. Biophys., 10, 15 I-158.

Schoch H., Schurch A.,Craseman E., 1964. Experiments on the energetic efficiency of frozen and dehydrated grass for the ruminants. "Energy metabolism". Proc. 3rd Symp. Troon, Scotland, May 1964,225 .

Tulloh N. M., Hughes J. W., Newth R. P., I965. Physical studies of the alimentary tract of grazing cattle. I. Measurement of volume of water in the reticulo-rumen. N.Z.J.Agric. Res., 8, 636-65I.

Waldo D. R., Miller R. W., OKamoto M., Moore L. A., I965. Ruminant utilization of silage in relation to hay, pellets and hay plus grain. 2. Rumen content, dry matter passage, and water intake. J. Dairy Sci., 48, I473-1480. 